\title{
Drug-Eluting Stent (DES) Induced Coronary Artery Aneurysm - A Case Report
}

\author{
A K Choudhury, M Akhtaruzzaman, M Khalequzzaman, S Hasem, T A Choudhury, N I Sharafat
}

Dept. of Cardiology, NICVD, Dhaka.

Keywords:
Coronary
artery
aneurysm,
DES.

\section{Introduction:}

Coronary artery aneurysms after coronary intervention are rare, the incidence of coronary artery aneurysms after DES implantation is low within the first 9 months, with a reported incidence of $0.2 \%$ to $2.3 \%$, a rate similar to that reported after bare-metal stent (BMS) implantation ( $0.3 \%$ to $3.9 \%)$ in the DES versus BMS randomized trials. Most "aneurysms" are in fact pseudoaneurysms rather than true aneurysms. ${ }^{1-4}$ Residual dissection and deep arterial wall injury (rupture or resection of the vessel media) caused by oversized balloons or stents, high pressure balloon inflations, atherectomy, and laser angioplasty have all been associated with coronary artery aneurysms after coronary intervention. ${ }^{1-3}$ Drug-eluting stents (DES), which locally elute antiproliferative drugs, can dramatically inhibit neointimal growth, thereby suppressing restenosis, ${ }^{5,6}$ but at the same time potentially causing coronary aneurysms due to other mechanisms, such as delayed re-endothelialization, inflammatory changes of the medial wall, and hypersensitivity reactions. ${ }^{7-10}$ These findings may be due to delayed healing secondary to the antiproliferative action of the eluted drug, cell necrosis and/or apoptosis from the antimetabolite effect of the drug, and hypersensitivity reactions to the drug/polymer mixture on the DES. ${ }^{7-9}$ Systemic administration of anti-inflammatory agents (glucocorticoids and colchicine) after stent implantation may be associated with a greater risk of aneurysm formation. ${ }^{11}$ However, the true incidence, clinical course, and treatment of coronary artery aneurysms after DES implantation remain largely unknown.

\section{Case Report:}

Mr. X, 60 years old non smoker, non alcoholic, diabetic, hypertensive businessman got admitted in NICVD with complaint of ischaemic chest pain on minimal exertion for 3 months. ECG was within in normal limit, ETT was positive and Echocardiogram showed Anterior wall hypokinesia with EF- 65\%. CAG showed significant long lesion in LAD. Direct stenting to LAD was done at the same setting with Promus Element $(2.75 \times 38 \mathrm{~mm}$, at 18 ATM). Whole

Address of Correspondence: Dr. Amal Kumar Choudhury, Department of Cardiology, National Institute of Cardiovascular Diseases, Dhaka, Bangladesh. E-mail: amal.choudhury@yahoo.com. 


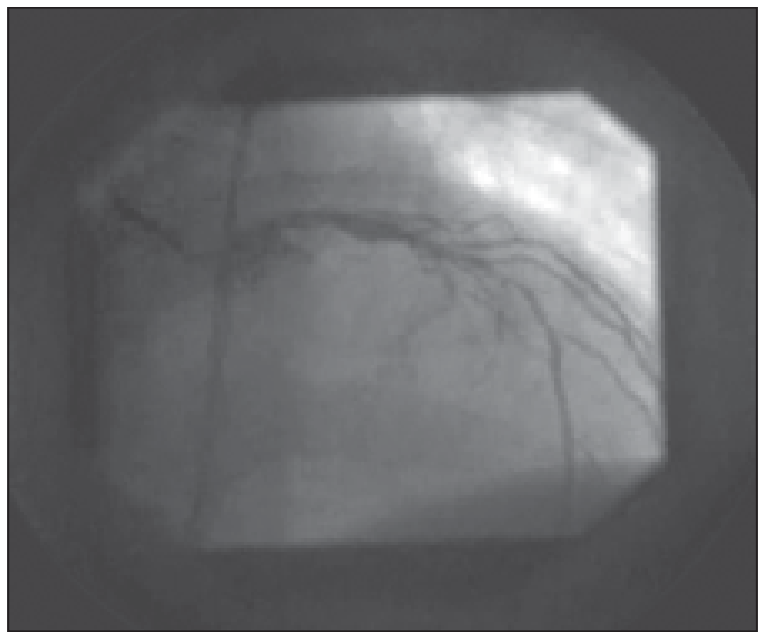

Fig.-1: CAG showing lesion in $L A D$

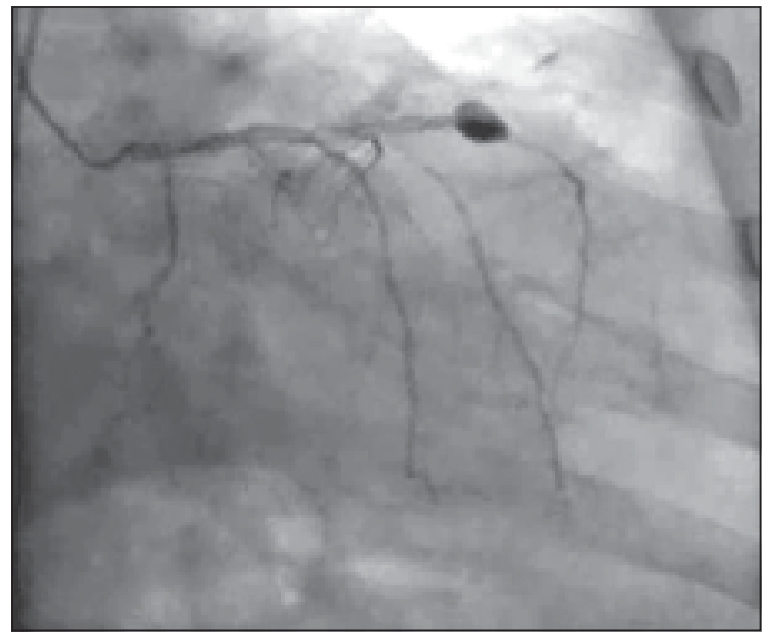

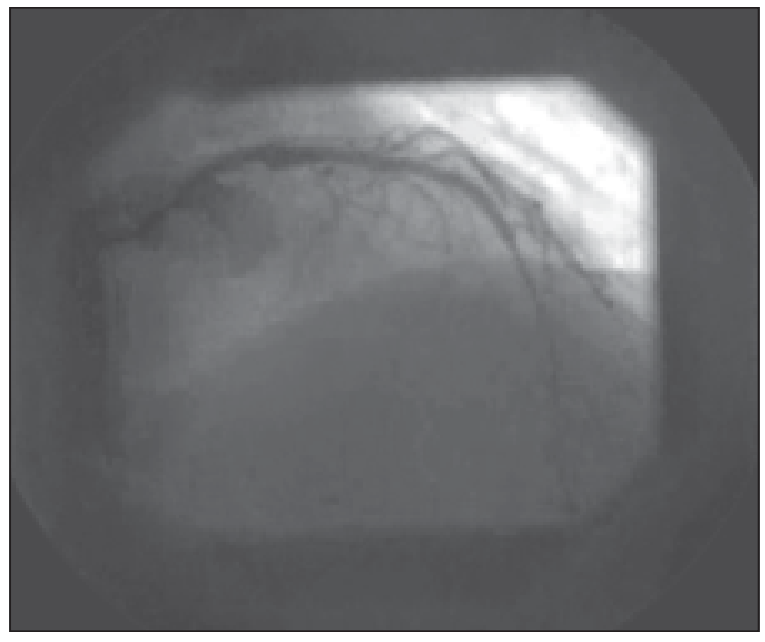

Fig.-2: After PTCA with Stenting

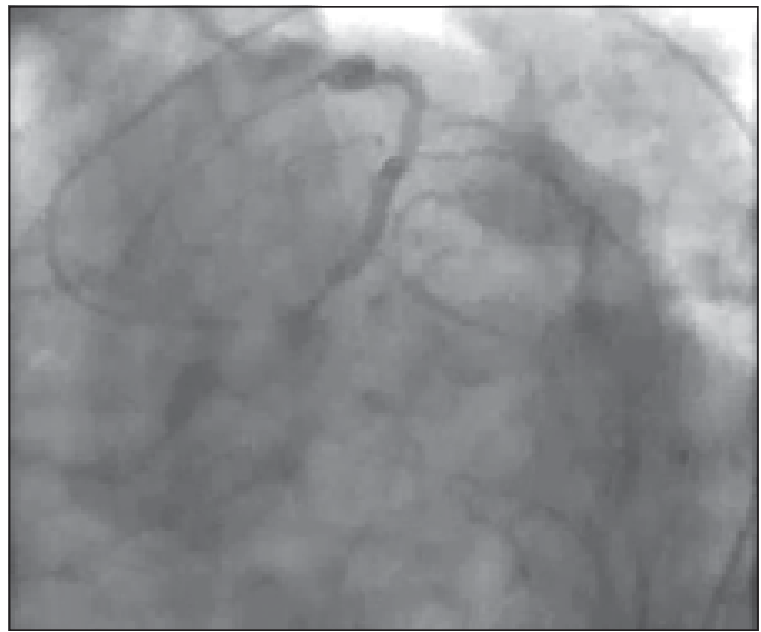

Fig.-3: Check CAG show coronary aneurysm at distal end of patent stent in LAD

procedure was uneventful and patient was discharged from hospital with double antiplatelet coverage. After 10 days of PCI patient got readmitted in hospital with complaints of chest discomfort with high grade fever for 2 days. ECG showed AMI ( Extensive Anterior) indicating involvement of LAD territory with strong suspicion of Sub Acute Stent Thrombosis (SAST). Streptokinase could not be given due to delayed arrival. Patient was treated conservatively with LMWH. Blood and urine culture was negative. Check CAG was done 7 days after readmission showing Patent stent in LAD with aneurysmal dilatation at the distal end of stent in LAD.

\section{Discussion:}

Coronary intervention-associated aneurysms usually are detected at the time of repeat angiography for recurrent symptoms or as part of routine angiographic follow-up as mandated by study protocols. Coronary angiography is the gold standard for the diagnosis of coronary aneurysms, which are defined as a luminal dilation $50 \%$ larger than that of the adjacent reference segment. ${ }^{3,11,12}$ However, coronary angiography provides only luminal information and cannot visualize other structures, including the layers of the arterial wall. Further discrimination between true aneurysms and pseudoaneurysms, as well as detection of the aneurysm entry site (sometimes caused by stent edge dissection or stent edge injury at acute bends during vessel movement), are important to optimally manage aneurysms after coronary intervention. Intravascular ultrasound (IVUS) has become the "gold standard" in providing 
critical diagnostic information to address these anatomic considerations in the evaluation of coronary aneurysms. ${ }^{13-15}$ Furthermore, other advanced coronary imaging techniques, such as computed tomography angiography, coronary magnetic resonance angiography, and real-time 3-dimensional echocardiography, also can be used as tools to detect and follow certain coronary aneurysms noninvasively, ${ }^{16-20}$ and computed tomography angiography may be the most effective of these in the milieu of metal stent artifacts. ${ }^{21}$ In addition to the mechanical risk factors for aneurysm formation that are observed with both BMS and DES, there are other potential mechanisms that may be specific to DES. Although inflammatory and allergic reactions to nickel and molybdenum have been reported after BMS implantation, ${ }^{22}$ the triggers for inflammatory and allergic reactions after DES implantation are more complex because DES consist of 3 components: the antirestenotic drug, the drug carrier vehicle (polymer), and the stent platform. In particular, the polymer carrier has been shown to provoke eosinophilic/ heterophilic infiltration and induce a marked inflammatory reaction of the arterial wall. $8,23,24$ In addition, delayed healing reactions in response to DES, such as incomplete endothelialization over DES struts, have been detected by invasive approaches (angioscopy and optical coherence tomography) as well as in autopsy studies. . $^{9,25,26}$ In short, the combination of physical trauma induced by stent implantation and specific biological reactions after DES implantation might together contribute to coronary aneurysm formation after DES implantation. In the DES versus BMS randomized trials, routine angiographic follow-up was performed in a large subset of patients at 6 to 9 months after the initial procedure. In this analysis, the incidence of coronary aneurysms was similar with DES compared with BMS. 1.1\% with DES and 0.8\% with BMS ${ }^{5,6,27-29}$. Three different types of aneurysms after DES or BMS implantation have been described. Type I aneurysm is a type of aneurysm that demonstrates rapid early growth with pseudoaneurysm formation detected within 4 weeks. ${ }^{30,31}$ This type is typically complicated by clinical pericarditis. Given the rapid time course of aneurysm formation, it is likely that arterial injury related to the procedure is the likely contributor to aneurysm formation in these cases rather than the chronic arterial response to the stent, polymer, and drug. The second kind of aneurysm described in the literature is that with a "subacute to chronic" presentation (type II) and is typically detected incidentally during angiography for recurrent symptoms or as part of protocol mandated follow-up (usually detected 6 months after the procedure). ${ }^{32-39}$ These aneurysms appear to have the most varied clinical presentations; some patients are asymptomatic, but some have complaints of angina. It seems more likely in this scenario that a chronic arterial response to a metal stent, polymer, and/or drug, may be the basis for aneurysm formation in this subtype. The final reported subtype in the published literature is mycotic or infectious in etiology (type III). ${ }^{40,41,42}$ Large mycotic aneurysms infected with Staphylococcus aureus after DES or BMS implantation have been reported. In these rare cases, patients typically present with systemic manifestations and fever as the result of bacteremia. Whether the local immunosuppressive effects of eluted drugs from stents tend to increase the incidence of these rare infectious aneurysms is unknown. Treatment of coronary aneurysms must be "individualized" considering the aneurysm size, expansion history, pathophysiology, and symptoms. For pseudoaneurysms detected by IVUS (type I) that are large at presentation (i.e., at least twice the reference vessel diameter) or show significant expansion over time, especially in the presence of symptoms, propose interventional or surgical treatment. Our threshold for treatment is lower for pseudoaneurysms than for true aneurysms, because of the presumed greater likelihood for rupture. For large true aneurysms (type II), again more than twice as large as the reference vessel diameter, especially with symptoms, we would also propose interventional or surgical treatment to avoid potential life threatening complications, regardless of stent type (BMS or DES). We propose immediate surgical therapy for any confirmed infected aneurysm (type III). Finally, long-term antithrombotic drug therapy, such as aspirin and clopidogrel, should be necessary to reduce the risk of stent thrombosis 
and distal embolism in patients with coronary artery aneurysms ${ }^{43,44}$. Clearly, there is no consensus regarding the treatment algorithm and our proposal is not based on prospective data, underscoring the need for further study of this relatively infrequent phenomenon.

\section{Conclusion:}

The clinical course of coronary artery aneurysms after DES implantation is variable. Some aneurysms naturally resolve, but some aneurysms can lead to life-threatening complications. Although the best treatment for coronary aneurysms after DES is controversial. Expanding pseudoaneurysms, infected aneurysms, and large, chronic (and expanding) aneurysms with symptoms should be treated.

\section{Conflict of Interest - None.}

\section{References:}

1. Bell MR, Garratt KN, Bresnahan JF, Edwards WD, Holmes DR Jr. Relation of deep arterial resection and coronary artery aneurysms after directional coronary atherectomy. J Am Coll Cardiol 1992;20:1474-1481.

2. Baumbach A, Bittl JA, Fleck E, et al. Acute complications of excimer laser coronary angioplasty: a detailed analysis of multicenter results. Coinvestigators of the U.S. and European Percutaneous Excimer Laser Coronary Angioplasty (PELCA) Registries. J Am Coll Cardiol 1994; 23:1305-1313.

3. Slota PA, Fischman DL, Savage MP, Rake R and Goldberg S. Frequency and outcome of development of coronary artery aneurysm after intracoronary stent placement and angioplasty. STRESS Trial Investigators. Am J Cardiol 1997;79:1104-6.

4. Condado JA, Waksman R,Gurdiel O, et al. Long-term angiographic and clinical outcome after percutaneous transluminal coronary angioplasty and intracoronary radiation therapy in humans. Circulation 1997;96:727-732.

5. Popma JJ, Leon MB, Moses JW, et al. Quantitative assessment of angiographic restenosis after sirolimuseluting stent implantation in native coronary arteries. Circulation 2004;110:3773-3780.

6. Stone GW, Ellis SG, Cox DA, et al. A polymer-based, paclitaxeleluting stent in patients with coronary artery disease. N Engl J Med 2004;350:221-231.

7. Virmani R, Liistro F, Stankovic G, et al. Mechanism of late in-stent restenosis after implantation of a paclitaxel derivate-eluting polymer stent system in humans. Circulation 2002;106:2649-2651.

8. Virmani R, Guagliumi G, Farb A, et al. Localized hypersensitivity and late coronary thrombosis secondary to a sirolimus-eluting stent: should we be cautious? Circulation 2004;109:701-705.
9. Joner M, Finn AV, Farb A, et al. Pathology of drug-eluting stents in humans: delayed healing and late thrombotic risk. J Am Coll Cardiol 2006;48:193-202.

10. Luscher TF, Steffel J, Eberli FR, et al. Drug-eluting stent and coronary thrombosis: biological mechanisms and clinical implications. Circulation 2007;115:1051- 1058.

11. Syed M and Lesch M. Coronary artery aneurysm: a review. Prog Cardiovasc Dis 1997;40:77-84.

12. Demopoulos VP, Olympios CD and Fakiolas CN, et al. The natural history of aneurysmal coronary artery disease. Heart 1997;78:136-141.

13. Maehara A, Mintz GS, Ahmed JM, et al. An intravascular ultrasound classification of angiographic coronary artery aneurysms. Am J Cardiol 2001;88:365-370.

14. Porto I, MacDonald S, Banning AP. Intravascular ultrasound as a significant tool for diagnosis and management of coronary aneurysms. Cardiovasc Intervent Radiol 2004;27:666-668.

15. Ge J, Liu F, Kearney P, et al. Intravascular ultrasound approach to the diagnosis of coronary artery aneurysms. Am Heart J 1995;130:765-771.

16. Achenbach S. Computed tomography coronary angiography. J Am Coll Cardiol 2006;48:1919-1928.

17. Weininger M, Meesmann M, Hahn D and Beissert M. Assessment of a left coronary artery aneurysm with 64channel multi-slice cardiac computed tomography. Eur J Cardiothorac Surg 2006;30:381-382.

18. Mavrogeni S, Papadopoulos G, Douskou M, et al. Magnetic resonance angiography is equivalent to $\mathrm{X}$-ray coronary angiography for the evaluation of coronary arteries in Kawasaki disease. J Am Coll Cardiol 2004;43:649-652.

19. Greil GF, Stuber M, Botnar RM, et al. Coronary magnetic resonance angiography in adolescents and young adults with kawasaki disease. Circulation 2002;105:908 -911.

20. Tsutsui JM, Martinez EE, Rochitte CE, Ramires JF and Mathias W Jr. Noninvasive evaluation of left circumflex coronary aneurysm by realtime three-dimensional echocardiography. Eur J Echocardiogr 2006;7: 75-78.

21. Degertekin M, Serruys PW, Tanabe K, et al. Long-term follow-up of incomplete stent apposition in patients who received sirolimus-eluting stent for de novo coronary lesions: an intravascular ultrasound analysis. Circulation 2003;108:2747-2750.

22. Koster R, Vieluf D, Kiehn M, et al. Nickel and molybdenum contact allergies in patients with coronary in-stent restenosis. Lancet 2000;356: 1895-1897.

23. Van der Giessen WJ, Lincoff AM, Schwartz RS, et al. Marked inflammatory sequelae to implantation of biodegradable and nonbiodegradable polymers in porcine coronary arteries. Circulation 1996;94: 1690-1697.

24. Finn AV, Nakazawa G, Joner M, et al. Vascular responses to drug eluting stents: importance of delayed healing. Arterioscler Thromb Vasc Biol 2007;27:1500-1510. 
25. Kotani J, Awata M, Nanto S, et al. Incomplete neointimal coverage of sirolimus-eluting stents: angioscopic findings. J Am Coll Cardiol 2006;47:2108 -2111.

26. Matsumoto D, Shite J, Shinke T, et al. Neointimal coverage of sirolimus-eluting stents at 6-month follow-up: evaluated by optical coherence tomography. Eur Heart $J$ 2007;28:961-967.

27. Colombo A, Drzewiecki J, Banning A, et al. Randomized study to assess the effectiveness of slow- and moderaterelease polymer-based paclitaxel-eluting stents for coronary artery lesions. Circulation 2003; 108:788-794.

28. Stone GW, Ellis SG, Cannon L, et al. Comparison of a polymer-based paclitaxel-eluting stent with a bare metal stent in patients with complex coronary artery disease: a randomized controlled trial. JAMA 2005; 294:1215-1223.

29. Dawkins KD, Grube E, Guagliumi G, et al. Clinical efficacy of polymer-based paclitaxel-eluting stents in the treatment of complex, long coronary artery lesions from a multicenter, randomized trial: support for the use of drugeluting stents in contemporary clinical practice. Circulation 2005;112:3306-3313.

30. Gupta RK, Sapra R and Kaul U. Early aneurysm formation after drugeluting stent implantation: an unusual lifethreatening complication. J Invasive Cardiol 2006;18: E140-142.

31. Zhang F, Qian JY and Ge JB. Rapid development of late stent malappositon and coronary aneurysm following implantation of a paclitaxeleluting coronary stent. Chin Med J(Engl) 2007;120:614-616.

32. Panja M, Basu S and Mondol S. A case of giant aneurysm following percutaneous coronary intervention. Indian Heart J 2005;57:731-733.

33. Nilsen DW, Melberg T, Larsen AI, Barvik S and Bonarjee V. Late complications following the deployment of drug eluting stents. Int J Cardiol 2006;109:398-401.
34. Kaul U, Gupta RK and Kachru R. Large coronary artery aneurysms following sirolimus eluting stent implantation. Heart 2005;91:234.

35. Anandaraja S, Naik N and Talwar K. Coronary artery aneurysm following drug-eluting stent implantation. $J$ Invasive Cardiol 2006;18:E66 -67.

36. Vik-Mo H, Wiseth $\mathrm{R}$ and Hegbom K. Coronary aneurysm after implantation of a paclitaxel-eluting stent. Scand Cardiovasc J 2004;38:349-352.

37. Kim HS, Park JH and Ko JK. Asymptomatic coronary artery aneurysm associated with paclitaxel eluting stent. Heart 2006;92:480.

38. Kim JW, Seo HS, Suh SY, Rha SW, Park CG and Oh DJ. Spontaneous resolution of neoaneurysm following implantation of a paclitaxeleluting coronary stent. Int $J$ Cardiol 2006;112:e12-13.

39. Bavry AA, Chiu JH, Jefferson BK, et al. Development of coronary aneurysm after drug-eluting stent implantation. Ann Intern Med 2007;146:230 -232.

40. Le MQ and Narins CR. Mycotic pseudoaneurysm of the left circumflex coronary artery: A fatal complication following drug-eluting stent implantation. Catheter Cardiovasc Interv 2007;69:508 -512.

41. Bouchart F, Dubar A, Bessou JP, et al. Pseudomonas aeruginosa coronary stent infection. Ann Thorac Surg 1997;64:1810-1813.

42. Liu JC, Cziperle DJ, Kleinman B and Loeb H. Coronary abscess: a complication of stenting. Catheter Cardiovasc Interv 2003;58:69-71.

43. Oyama N, Urasawa K and Kitabatake A. Detection and treatment of coronary artery pseudoaneurysms following coronary stent deployment. J Invasive Cardiol 2004;16: $521-523$.

44. Swaye PS, Fisher LD, Litwin P, et al. Aneurysmal coronary artery disease. Circulation 1983;67:134-138. 